\title{
Análisis de la percepción de los servicios municipales en la comunidad anzoatiguense, Venezuela
}

\author{
Núñez, Linda ${ }^{\dagger}$ * \\ Rodríguez, Daysi ** \\ Cáceres, Alfonso ***
}

\section{Resumen}

Este artículo tiene como es analizar la percepción de la comunidad anzoatiguense en relación a la prestación de los servicios municipales, como parte de la gestión pública por resultados; con un aporte hacia una primera aproximación metodológica como estudio de opinión en la evaluación de las políticas públicas municipales. En base a la gestión de los alcaldes, utilizando indicadores de percepción administrativa, de acuerdo a las competencias que confiere sobre el particular la Constitución de la República Bolivariana de Venezuela en la geografía de estudio de los municipios: Sotillo, Urbaneja, Bolívar, Guanta, Peñalver, del estado Anzoátegui, Venezuela; y a través de la opinión de la prestación de los servicios públicos, se jerarquizaron los indicadores a través de un análisis de componente principales (varimax) de factores rotados. El levantamiento de la información se realizó durante el mes de septiembre 2008 y la información obtenida se procesó con el software estadístico SPSS 15. El enfoque metodológico aplicado permitió precisar las prioridades de la ciudadanía a las políticas implementadas por los municipios de acuerdo a la ley. Nuestros hallazgos evidencian que la cuidadania de los municipios Sotillo, Urbaneja y Guanta, en su evaluación, prioriza los servicios de limpieza de aceras y calles y de recolección de basura; mientras que los habitantes de Bolívar y Guanta le dieron mayor importancia a las políticas públicas relacionadas con: recreación, cultura y deporte.

Palabras clave: Análisis de gestión, servicios municipales, percepción ciudadana.

Recibido: 05-11-09. Aceptado: 05-03-10

* Doctora en Ciencias, mención Ciencias Políticas, Universidad Central de Venezuela (2008). Directora de la Escuela de Ciencias Administrativas de la UDO, Núcleo de Anzoátegui. Profesora Titular a dedicación exclusiva. Investigador acreditado en el Programa de Promoción al Investigador. E-mail: lync61@gmail.com

** Magíster Scientiarum en Ciencias Administrativas mención Finanzas. Premio de Promoción al Investigador (PPI) Nivel I con el Nº. 9267. Investigador Activo Nivel IV en Comisión de investigación, UDO, Núcleo de Anzoátegui. Docente de la Universidad de Oriente (UDO) núcleo de Anzoátegui.

*** Doctorando en Gerencia. Docente de la Universidad de Oriente, núcleo de Anzoátegui. Coordinador de la comisión de Investigación del núcleo de Anzoátegui. Investigador acreditado en el Programa de Promoción al Investigador. 


\title{
Analysis of the Perception of Municipal Services in the Anzoátegui Community, Venezuela
}

\begin{abstract}
The purpose of this article is to analyze the perception the Anzoátegui community has related to municipal services provided as part of public management by results, contributing to an initial methodological approach as an opinion study for evaluating municipal public policies. Based on management by the mayors, according to powers conferred by the Constitution of the Bolivarian Republic of Venezuela in the municipalities of Sotillo, Urbaneja, Bolivar, Guanta Penalver, State of Anzoategui, Venezuela, using administrative perception indicators, and through opinions regarding the provision of public services, the indicators were prioritized using a principal component analysis (varimax) of rotated factors. Information was collected in September, 2008, and processed with statistical software SPSS 15. The methodological approach used made it possible to determine citizen priorities for policies implemented by the municipalities according to law. Findings show that citizens of the Sotillo, Urbaneja and Guanta municipalities prioritize street and sidewalk cleaning and garbage collection in their evaluations, while residents of Bolivar and Guanta gave greater importance to public policies related to recreation, culture and sport.
\end{abstract}

Key words: Management analysis, municipal services, citizen perception.

\section{Introducción}

En los últimos tiempos, se ha considerado la conveniencia de la evaluación y participación social y ciudadana basada en la teoría de la gerencia pública, específicamente en el análisis de gestión por resultados de las políticas gubernamentales. Cardozo (2005) considera que en América Latina se cuenta con avances muy restringidos en materia de evaluación de la acción pública y participación social. México, por ejemplo, a pesar de que ha realizado progresos importantes a partir de 2001, la calidad alcanzada aún enfrenta problemas diversos y no se correlaciona con el presupuesto invertido en su realización. La aludida autora reflexiona que es urgente que las universidades de América Latina formen evaluadores con conocimiento del enfoque partici- pativo, especialmente en el caso de programas que apoyen el desarrollo económico-social, con carácter sustentable.

Los medios de comunicación venezolanos informan continuamente de los acontecimientos que afectan negativamente a la sociedad, evidenciando los problemas que se presentan en los municipios, situación que debería ser resuelta con políticas públicas adecuadas en la prestación de los servicios ofrecidos por los alcaldes, de acuerdo a lo conferido en las leyes venezolanas, tomando en consideración una nueva forma de gobierno, igualmente contempladas en la Constitución Nacional y demás leyes, al incorporar la opinión de la comunidad para el diseño de dichas políticas en busca de lograr el bienestar de la sociedad representada por dichos actores municipales. Sin embargo la falta de evaluación de la ges- 
Análisis de la percepción de los servicios municipales en la comunidad anzoatiguense Núñez, Linda; Rodríguez, Daysi y Cáceres, Alfonso

tión de los alcaldes imposibilita evidenciar el impacto de las políticas implementadas, igualmente dificulta evidenciar la satisfacción de comunidad en el disfrute de los servicios.

En este sentido el estudio realizado por Fernández, et al. (2008:394) en Venezuela, evidenció que el estado venezolano ha promulgado leyes y ha formulado políticas con el propósito de disminuir la exclusión social de las comunidades. En este sentido, para los autores, los lineamientos en materia informacional no se ajustan a la realidad de los municipios lo que dificulta el alcance de los planes que se emprendan. Adicionalmente, concluyeron que la participación ciudadana sirve de apoyo al proceso de la gestión pública local.

En este orden de ideas, el propósito de este estudio es analizar la percepción de la comunidad anzoatiguense en relación a la prestación de los servicios municipales, como parte de la gestión pública por resultados; con un aporte hacia una primera aproximación metodológica como estudio de opinión en la evaluación de las políticas públicas municipales. Se tomaron en este trabajo los programas establecidos en los municipios: Simón Bolívar (Barcelona), Juan Antonio Sotillo (Puerto La Cruz) y Diego Bautista Urbaneja (Lechería), Guanta (Guanta), Fernando de Peñalver (Puerto Píritu), del estado Anzoátegui, Venezuela, de acuerdo al marco legal que rige dicha gestión, con mediciones realizadas a través de encuestas de opinión y preguntas sobre indicadores de gestión, que intentan capturar la realidad social de los municipios mencionados. Se hizo una revisión literaria a la gestión pública por resultados, combinada con evaluación, participación ciudadana e indicadores de gestión y percepción pública para lograr el objetivo de la investigación.

Ante este panorama surge la siguiente interrogante: ¿cómo percibe la comunidad el desempeño de la gestión municipal? Punto de interés que se abordará en este estudio. Primeramente se realizará un abordaje descriptivo en lo atinente a la gestión por resultados y evaluación. Seguidamente se dará una explicación de los indicadores de gestión y por último se tomará en cuenta la importancia de la participación ciudadana y la percepción pública para formar así una triada en aras de dar un aporte a la gerencia de la administración pública, mediante nuevas formas de análisis de gestión por resultados.

La metodología utilizada en este trabajo para el análisis de la prestación de servicios por parte de la comunidad anzoatiguense, se fundamentó en primer lugar, en la utilización de indicadores de percepción administrativa de los servicios que prestan los municipios. Para esto se revisó las competencias de los mismos, establecidas en el Art. No. 178 de la Constitución de la República Bolivariana de Venezuela y el Art. No. 4 de Ley Orgánica del Poder Público Municipal.

En segundo lugar, la captura de la información se basó en los postulados de la satisfacción del ciudadano y eficiencia en la prestación de los servicios públicos. Estos criterios considerados dan una imagen de la prestación de servicios y la calidad de los mismos, además arroja el ranking de prioridad a los alcaldes para la asignación de los recursos y la mejora de su política. Como técnica se usó la encuesta para ponderar la opinión de la colectividad sobre la calidad de los servicios 
públicos, en vías de identificar la participación comunitaria en función de las demandas colectivas versus la gestión de los servicios públicos locales.

Con la finalidad de analizar la opinión de los encuestados se aplicó un instrumento (cuestionario) utilizando el Muestreo Aleatorio Simple (MAS) con una confiabilidad el $95 \%$ y un error de muestreo de 0,05. El levantamiento de la información se realizó durante el mes de septiembre 2008 en los municipios: Sotillo, Urbaneja, Guanta, Bolívar y Peñalver, del estado Anzoátegui, Venezuela.

\section{Percepción Pública}

Los estudios de percepción pública y participación ciudadana en la evaluación de las políticas públicas en las entidades locales, han venido teniendo una presencia mayor a lo largo de las últimas décadas. Sin embargo, aún cuando pueda postularse la universalización del conocimiento científico y tecnológico, es indudable que su recepción, apropiación y empleo son procesos socialmente situados y sujetos tanto a las especificidades culturales de cada sociedad como a las situaciones sociales históricas y concretas de éstas. Estas especificidades se observan en distintos órdenes institucionales de la sociedad, teniendo en cuenta la relación de la vida cotidiana de las comunidades y las instituciones gubernamentales.

Los estudios tradicionales de indicadores de percepción pública y cultura científica han recibido el respaldo de instituciones públicas responsables de la formulación de políticas y gestión de la ciencia, a partir de las cuales se ha ido desarrollando, desde hace unos treinta años, un área de trabajo interdisciplinario que permitió confeccionar metodologías de encuestas y análisis de datos, básicamente en los países desarrollados (Polino et al., 2003).

Ahora bien, diversas teorías de percepción han sido la clave en el desarrollo de estudios de percepción pública y de opinión. Al respecto, Robbins (1998) considera la percepción "como un proceso mediante el cual, los individuos organizan e interpretan sus impresiones sensoriales con el fin de darle significado a su ambiente", similarmente Lambin (1995) la analiza como [...] proceso por el cual un individuo selecciona e interpreta la información a la cual está expuesto. Los individuos tendrán, pues, en general, percepciones distintas de una misma situación, porque la atención es selectiva. La percepción tiene una función reguladora, en el sentido que filtra la información. De un modo singular Wittig (1979) agrega "los procesos sensoriales simplemente informan acerca de los estímulos ambientales; la percepción traduce estos mensajes sensoriales en forma comprensible".

A partir de estas teorías clásicas los investigadores buscan explicar los fenómenos susceptibles en los estudios de percepción pública, basados también en la teoría de la percepción denominada Gestaltica (1920), que indica que "la percepción debe estudiarse no analizando unidades aisladas como las sensaciones simples, sino tomando en cuenta configuraciones globales" (Stanley et al., 1999).

Sin embargo, existe una serie de factores que ayudan a dar forma o a distorsionar la percepción, los que pueden residir en el perceptor, cuando un individuo observa un blanco, tratará de inter- 
Análisis de la percepción de los servicios municipales en la comunidad anzoatiguense Núñez, Linda; Rodríguez, Daysi y Cáceres, Alfonso

pretar lo que está viendo, pero esta interpretación estará condicionada por las características personales del perceptor, donde destacan la actitud, los motivos insatisfechos, los variados intereses personales, sus expectativas así como la experiencia, que también es un agente modificador de su apreciación. Ante tales apreciaciones, es conveniente considerar la teoría de la espiral del silencio que declara "que es menos probable que un individuo dé su opinión sobre un determinado tema entre un grupo de personas si siente que es parte de la minoría, por miedo a la represión o aislamiento por parte de la mayoría" (Anderson, 1996 y Miller, 2005).

En síntesis, estas teorías en su conjunto permitieron elaborar un instrumento de indagación (encuesta) sobre la percepción de la comunidad anzoatiguense en relación a la prestación de los servicios municipales.

\section{Gestión pública por resultados y la evaluación}

La gestión pública por resultados conlleva a la asignación de los fondos sobre la base de los cambios en el bienestar social que este genera, esto implica cambiar las estructuras de toma de decisiones de asignación presupuestal. Involucra del mismo modo, la implementación de cambios en la forma de programar o planificar el gasto, también implica la medición del desempeño a través de indicadores, la evaluación sobre la base de tales indicadores y la generación de incentivos y flexibilidades para que los gerentes públicos puedan tomar decisiones de manera ágil. Requiere también de un cambio en la racionalidad del control social y la vigilancia por parte de la sociedad civil.

La gestión por resultados crea la necesidad de buscar la calidad de los servicios ofrecidos por el Estado e incorporar las expectativas de los ciudadanos en el diseño e implementación de los productos y bienes públicos. Ante esto, busca que la administración de los recursos públicos se centre en el cumplimiento de las acciones estratégicas definidas en el plan de gobierno, en un período de tiempo determinado. De esta forma, permite a los gobernantes gestionar y evaluar las acciones de las organizaciones del Estado con relación a las políticas públicas definidas para atender las demandas de la sociedad (Oszlak, 2003). Se produce un inédito consenso acerca de sus bondades, en el campo de la reforma del estado. Permite contar con instituciones realmente eficaces en la resolución de los problemas presentados mediante la formulación de políticas públicas bien diseñadas e implementadas, y la corrección de dicha política en los momentos de que se evidencia que no están logrando la solución del problema y por ende lograr el fin último de toda política como lo es el bienestar de la sociedad.

En el caso de los municipios, observamos como la gestión por resultados está adquiriendo gran importancia en muchos países del mundo y particularmente de América Latina, debido a cambios y transformaciones en las prácticas administrativas orientadas hacia una nueva visión de la administración pública. La calidad en las actuaciones de las entidades públicas ha pasado a ocupar un lugar prioritario enmarcado en un nuevo modelo de gestión 
pública (Blanco et al., 2007). La gestión pública tradicional ha venido padeciendo, por muchos años, de distintas debilidades, que probablemente explican la incapacidad de los entes públicos para desarrollar apropiados procesos de provisión de servicios a favor de la población, la cual reclama en el marco de las libertades democráticas, mayor cantidad y calidad de servicios, a fin de atenuar los factores críticos limitantes de las condiciones de vida de la población (Acevedo, 2005).

La esencia de la gestión municipal es atender y satisfacer las solicitudes y demandas de la comunidad, traducidas en bienes y servicios públicos. Esto hace necesario superar la desvinculación existente entre la administración pública y las demandas sociales; por lo tanto, es preciso mejorar los canales de comunicación con los beneficiarios directos e indirectos de los programas establecidos y que se mejore los procesos internos para generar soluciones más eficientes. Es indispensable tomar en cuenta la valoración de la gestión municipal, dado que la evaluación es parte esencial del proceso de análisis de políticas públicas, hace posible la asignación de responsabilidades, considerado el corazón del sistema de gestión pública. Además de ello, permite, entre otras cosas, suministrar información acerca del desempeño de la política, tener insumos adecuados para la redefinición del problema y la reformulación de la política, así como la adopción de políticas alternativas (Dunn, 2004). Esto requiere considerar en detalle procesos de conceptualización y valoración empírica necesarios para enfrentar sistemáticamente problemas públicos para emprender una buena gestión.
En este sentido White (1994), hace énfasis en un enfoque múltiple como metodología de análisis. Este enfoque implica una concepción práctica del análisis político, en el sentido aristotélico de praxis como buena acción de gobierno y cuyo principal objetivo es la solución de problemas públicos mediante la aplicación sistemática de conocimientos y múltiples técnicas de estudio; ofrece además, información válida, confiable y contribuye al esclarecimiento de los valores que sustentan la selección de objetivos, fines y la posibilidad de aplicar otros métodos del análisis político. Seguido además, de una idónea evaluación de las políticas públicas en el logro de comprender muchas de las actuaciones y acciones en la ciudadanía con una valoración sistemática contribuyente al buen desempeño del programa (Weiss, 1998).

Ahora bien en cuanto a las técnicas de evaluación, tenemos que una de las más utilizadas, entre otras, es tomar en cuenta la opinión de la comunidad usando muestras de encuestas para evaluar la calidad de los servicios que ellos reciben de sus gobiernos. Esta técnica es apoyada por Langseth et al. (1996) del Instituto de Desarrollo Económico del Banco Mundial quienes dan herramientas de evaluación de gestión a través de las encuestas de opinión, utilizando preguntas de indicadores de gestión.

Pulsar a la sociedad permite obtener y difundir información sobre la opinión pública acerca de un programa, hace visible el apoyo social silencioso y facilita el proceso (Saltó et al., 2006). Además de ello, los sondeos de opinión pueden dar mayor acceso a la interrelación entre la opinión de los habitantes y las políticas aplicadas; por 
Análisis de la percepción de los servicios municipales en la comunidad anzoatiguense Núñez, Linda; Rodríguez, Daysi y Cáceres, Alfonso

otro lado, ayuda a que los funcionarios del gobierno puedan dar respuestas más adecuadas a las exigencias de la sociedad (Núñez, et al., 2007) y en el mejor de los casos hacer los correctivos a tiempo de las deficiencias del programa.

\section{Indicadores de gestión}

En los párrafos anteriores mencionamos la importancia de la valoración ya que permite suministrar información adecuada del desempeño de la política y el adecuado cumplimiento de las responsabilidades del ente público, es por ello que es imprescindible dotarse de buenos indicadores de gestión que nos puedan suministrar esta información.

Los indicadores de gestión no son más que una unidad de medida que permite el seguimiento y evaluación de los programas establecidos por los gobiernos, y, dado que los recursos provienen de una serie de contribuciones y/o impuestos obligatorios de diferentes sectores tanto públicos como privados para ser utilizados en los programas de servicios, establecidos de acuerdo a la Constitución Nacional, estos deben valorarse en función de la contribución de la satisfacción de las necesidades colectivas y el grado de cumplimiento de los objetivos (Guinart, 2003). Del mismo modo, los indicadores de gestión motivan al gerente público evaluarse según sus resultados alcanzados y a la ciudadania participar a través de la ponderación de la adecuada implementación de la política.

Bossi (2001) da una variada explicación de la clasificación de los indicadores según el objeto a medir siguiendo los lineamientos de indicadores de ges- tión para las entidades públicas de la Asociación Española de Contabilidad y Administración de Empresas. Recogiendo estos argumentos y dada la combinación entre gestión pública, gerente público, indicadores de gestión, evaluación y participación ciudadana, es importante el desarrollo del conjunto de indicadores que comprenda las diferentes dimensiones de la misma. En el caso que amerita para este estudio, se puede buscar dichas dimensiones, de acuerdo a la calidad de los servicios, desde la óptica del usuario. Por esta razón, conviene que se ponga énfasis en el enfoque orientado hacia el cliente. Dicho enfoque, según Guinart (2003) debe estar caracterizado por lo deseable y/o esperado de la administración pública. En este caso la mencionada autora da las siguientes caracteristicas: que sea comprensible para el ciudadano; que sea espacial, temporal y materialmente accesible; que aporte soluciones y que permita la participación del ciudadano en la determinación de necesidades administrativas que le afecten. Además hemos considerado las condiciones del indicador para la gestión pública, con un aporte de información imprescindible para informar, controlar, evaluar y tomar decisiones (relevancia), que no permita interpretaciones contrapuestas y ambigüedades (inequívoco), con un concepto claro y adecuado en el tiempo (pertinencia) y por último que cumpla con los criterios de objetividad, accesibilidad, sensibilidad y precisión.

\section{Participación Ciudadana}

La participación ciudadana es parte esencial de un sistema democrático y 
crea condiciones para el pleno desarrollo. El desafío en este ámbito requiere de fortalecer y construir herramientas que permitan un verdadero intercambio entre la sociedad civil y el poder establecido, por lo tanto es de gran importancia generar los espacios que contribuyan a que los derechos y deberes institucionalmente establecidos sean efectivamente reconocidos y ejercidos.

Para Bracho (2001) con esta forma de gobierno predomina la idea de considerar la participación como un instrumento fundamental para mejorar la capacidad de gobierno ya que además de ser un derecho, es un instrumento de optimación de la gestión pública. Ello traduce en la necesidad de dar respuesta a las exigencias del colectivo según la naturaleza de cada municipio y sin desnaturalizar su esencia (Matheus, 2006). La participación ciudadana ofrece un cauce que permite el control de las decisiones que las autoridades administrativas adoptan. Esto cobra fuerza en las administraciones públicas modernas, las cuales experimentan una crisis de legitimación democrática y permite entender la gestión pública de una manera más amplia, como las acciones de administrar, gestionar o hacer diligencias que conduzcan al logro de un asunto público. Bajo esta concepción, Morales et al. (2006) consideran que la participación ciudadana se relaciona íntimamente con la política pública y la administración pública, buscando incorporar la acción ciudadana a los procesos de decisión y operación del gobierno dentro de la referida gestión. Por tal motivo para los autores la participación ciudadana tiene varias fases que buscan eslabonarse y llegar a formar un continuum, ta- les como la información, la consulta, el seguimiento y control.

En este sentido, Escobar (2004: 97-108) encontró en su estudio que la participación ciudadana en America Latina, se advierten algunas posibilidades de ejercicio y constitución de ciudadanía, toda vez que comienzan a profundizarse en un número importante de países de la región procesos de descentralización más autónomos y el surgimiento de movimientos sociales y políticos de nuevo tipo, que comienzan a redefinir la relación con el Estado y el sistema político, a pesar de que existen condiciones de fragmentación social y política de vastos sectores de las sociedades latinoamericanas por deficiencias del Estado en cuanto a procesos de descentralización y desconcentración, así como en el déficit de participación política como expresión de la precariedad en la construcción de actores colectivos y sujetos.

Por su parte Pintaudi (2005) reflexiona, en su estudio realizado en Brasil, sobre la existencia de un dilema con la participación ciudadana con la llamada democracia participativa, ya que todo eso presupone ciudadanos informados y actuantes con influencia en la formulación y acompañamiento de políticas acordadas en conjunto, poder público y sociedad civil. La autora considera que para que pueda funcionar esta forma de gobierno, el mismo debe ser completamente transparente, informando todas sus acciones. Situación que no es fácil en la práctica, porque eso conlleva a que haya una buena formación del ciudadano y unos medios de comunicación que no informen según sus intereses. Cuestionando así junto con Sartori (2003:108) de qué ma- 
Análisis de la percepción de los servicios municipales en la comunidad anzoatiguense Núñez, Linda; Rodríguez, Daysi y Cáceres, Alfonso

nera el pueblo está capacitado para ejercer el poder. Este autor afirma que es necesario considerar la noción de opinión pública y la cuestión de la información. Montecinos (2006:205) concluye que en Chile a pesar de lo centralizado que resulta ser el diseño institucional subnacional, la participación ciudadana en la gestión municipal, no estuvo determinada necesariamente por incentivos institucionales gubernamentales del nivel central, sino más bien por aspectos políticos, endógenos y locales, los cuales implicaron la adopción de una función deliberadora de la municipalidad.

En Venezuela, este intercambio de ideas y experiencias se hace a través de los consejos locales de planificación, el cual se expresa en el Art. No. 2 de la Ley de los Consejos Locales de Planificación Pública, según la cual expone que los mismos son el órgano encargado de la planificación integral del gobierno local, cuyo propósito fundamental consiste en lograr la participación, el protagonismo de las comunidades organizadas, para lo cual deberán promover y planificar en función de las características propias de cada municipio, en atención a las condiciones de población, y la Ley de Consejos Comunales, estableciendo que los mismos (Art. No. 2) son instancias de participación, articulación e integración entre las diversas organizaciones comunitarias, grupos sociales y los ciudadanos y ciudadanas, que permiten al pueblo organizado ejercer directamente la gestión de las políticas públicas y proyectos, todo esto conforme a los lineamientos de la Constitución Nacional. Dentro de esta perspectiva, se puede decir que la creación de los Consejos Comunales, es una representación evidente de la democracia participativa y protagónica que busca el desarrollo sustentable de las comunidades, ya que con su constitución la sociedad es protagonista de su propio desarrollo al detectar los problemas que existen en el sector donde habitan para así analizarlos y adelantar acciones en pro de su solución (Marcano et al., 2008).

\section{Análisis comparativo de la gestión por resultados de los municipios en estudio}

La muestra probabilística de 2000 adultos tomadas en los municipios en estudio está conformada en su análisis descriptivo de la siguiente manera: sexo (45,4\% masculino, $54,6 \%$ femenino); edad (< de 20 años 26,\%, < de 35 años $41,4 \%$, $\geq$ de 35 años 32,6\%); ocupación (Estudiante 30,8\%, Empleado 41,9\%, Desempleado 7,2\%, Ama de casa $12 \%$, Otros 8,1\%); Estado civil (Soltero 53,7\%, Casado $35,10 \%$, Otro $11,2 \%$ ) y Reside en el Municipio (Sí 91,6\%, No 8,4\%).

Para medir la confiabilidad del cuestionario se utilizó el modelo el Alfa de Cronbach con valores superiores de 0,5 que demuestran la confiabilidad del mismo $(0,761)$. Inicialmente, el instrumento se diseñó con 20 ítems pero al efectuar la validez de constructo con la colaboración de expertos en la materia se quedó estructurado con siete (07) ítems que captan la percepción de los ciudadanos en cuanto a la calidad de la prestación de los servicios por parte del municipio. Con respecto a la validación se utilizó la técnica de análisis de factores mediante el método de extracción de componentes principales, ejecutándose como pruebas iní- 
ciales: la esfericidad de Bartlett y la adecuación de la muestra de Kayser-MeyerOlkin (KMO) con valores para la primera de $p<0,05$ y KMO $\geq 0,5$. La varianza acumulada explica por lo menos el $50 \%$ de los componentes principales (Gorsuch, 1997:68). Podemos inferir que el cuestionario se ajusta a los objetivos planteados en el estudio, en vista que la prueba de esfericidad del Bartlett arrojó un valor de 0,0001 y el KMO dio 0,816. A la solución factorial se le aplicó una rotación octogonal (varimax) para tener mayor claridad sobre los ítems que formaban parte de cada componente, considerándose los coeficientes en cada componente a partir de 0,400. Para este estudio los componentes que mayor aporte dan a la investigación son los componentes 1 y 2 con unos autovalores iníciales de 2,974 y 0,945 respectivamente y con un porcentaje de la varianza de 42,484\% (componente 1) y $13,504 \%$ (componente 2 ).
Tanto la medida de adecuación muestral del Kaiser-Meyer-Olkin (significativo si $\mathrm{KMO} \geq 0,5$ ) como la prueba de esfericidad de Bartlett (significativo si $p<0,05$ ) presentan valores que confirman la conveniencia del análisis factorial. El análisis factorial extrajo dos componentes principales (Tabla 1).

La Tabla 2 muestra al primer componente compuesto por las variables de percepción por parte de la comunidad en la prestación de los servicios por parte del municipio en cuanto a: mantenimiento de las calles y aceras $(0,75)$, servicio de recolección de basura $(0,72)$, programas de actividades deportivas y culturales $(0,65)$, áreas de esparcimiento y recreación $(0,64)$, servicio de cloacas y desagües $(0,62)$. Este componente captura parte de las responsabilidades del municipio establecidas en la Constitución Nacional. Con un porcentaje de la varianza de $42,48 \%$. El segundo componente captura la parte

Tabla 1

Matriz de prueba de adecuaciones de muestra y procedimiento

\begin{tabular}{cccccc}
\hline Grupo & Factores & Autovalor & \% varianza & KMO & P de Bartlett \\
\hline \multirow{2}{*}{ General } & Componente 1 & 2,97 & 42,484 & 0,816 & 0,0001 \\
& Componente 2 & 0,95 & 13,504 & & \\
Sotillo & Componente 1 & 2,88 & 41,160 & 0,815 & 0,0001 \\
& Componente 2 & 0,97 & 13,868 & & \\
Urbaneja & Componente 1 & 2,458 & 35,121 & 0,726 & 0,0001 \\
& Componente 2 & 1,122 & 16,031 & & \\
Guanta & Componente 1 & 2,471 & 35,293 & 0,738 & 0,0001 \\
& Componente 2 & 1,086 & 15,512 & & \\
Bolívar & Componente 1 & 2,487 & 35,526 & 0,744 & 0,0001 \\
& Componente 2 & 1,061 & 15,158 & & \\
Peñalver & Componente 1 & 3,113 & 44,468 & 0,793 & 0,0001 \\
& Componente 2 & 1,023 & 14,615 & & \\
\hline
\end{tabular}

Fuente: Elaboración propia. 
Análisis de la percepción de los servicios municipales en la comunidad anzoatiguense Núñez, Linda; Rodríguez, Daysi y Cáceres, Alfonso

Tabla 2 Matriz de componentes rotados(a)

\begin{tabular}{lcc}
\hline & \multicolumn{2}{c}{ Componente } \\
\cline { 2 - 3 } & $\mathbf{1}$ & $\mathbf{2}$ \\
\hline Calles-Aceras & 0.748 & 0.200 \\
Basura & 0.716 & 0.180 \\
Deporte-Cultura & 0.650 & 0.006 \\
Recreación & 0.636 & 0.176 \\
Cloacas-Desagües & 0.617 & 0.262 \\
Seguridad & 0.044 & 0.912 \\
Policía & 0.424 & 0.674 \\
\hline
\end{tabular}

Fuente: Elaboración propia.

de seguridad del municipio $(13,50 \%$ de la varianza) representado por la variables de percepción de la seguridad $(0,91)$ y la actuación de la policía $(0,67)$.

Los componentes principales capturan todas las variables de estudio en todos los municipios de la zona nororiental de estado Anzoátegui, Venezuela, tal como lo refleja la Tabla 3. En el Municipio Juan Antonio Sotillo los habitantes priorizan las políticas gubernamentales relacionadas con: mantenimiento de calles y aceras $(0,824)$, servicio de recolección de basura $(0,789)$, servicio de cloacas y desagües $(0,668)$ y las actividades deportivas y culturales $(0,417)$, agrupadas en el primer componente. En el Municipio Urbaneja los resultados, en el primer componente, son similares al municipio Sotillo, es decir la colectividad de Urbaneja le da como primera importancia a las mismas variables acentuadas en el municipio Sotillo, exceptuando las políticas de deporte y cultura. En el municipio Guanta, de los siete ítems incluidos en la encuesta, cinco quedaron aglutinados en el primer componente re- presentado por: mantenimiento de las calles y aceras $(0,791)$, servicio de recolección de basura $(0,728)$, (similar al municipio Sotillo y Urbaneja), recreación $(0,550)$, servicio de cloacas y desagües $(0,525)$, deporte y cultura $(0,595)$. En los municipios Bolívar y Peñalver, el comportamiento de la opinión de estas comunidades, son similares, es decir, el componente 1 representado por: recreación $(0,713)$, deporte y cultura $(0,685)$, calles y aceras $(0,641)$ y basura $(0,602)$, para Bolívar y para el municipio Peñalver: deporte y cultura $(0,809)$, recreación $(0,788)$, cloacas y desagües $(0,618)$.

\section{Conclusiones}

En enfoque metodológico aplicado permitió precisar las prioridades que da la ciudadanía Anzoatiguense a las políticas implementadas por los municipios de acuerdo a la Constitución Nacional y leyes orgánicas municipales. Los indicadores de gestión utilizados en las encuestas de opinión se perciben como una oportunidad para medir la evolución de los programas establecidos por los gobiernos tal como lo establece Guinart (2003) y Bossi (2001) en sus estrategias de gestión.

El análisis de la gestión local tomando en cuenta la opinión de la comunidad de los municipios Sotillo, Urbaneja, Bolívar, Guanta, Peñalver, del estado Anzoátegui, Venezuela, representa una técnica que debería utilizar los gobernantes en forma recurrente para evaluar la calidad de los servicios que se le otorgan a la dicha comunidad, tal como lo establece Langseth et al. (1996). Se evidencia como los municipios Sotillo, Urbaneja y 
Revista Venezolana de Gerencia, Año 15, No. 49, 2010

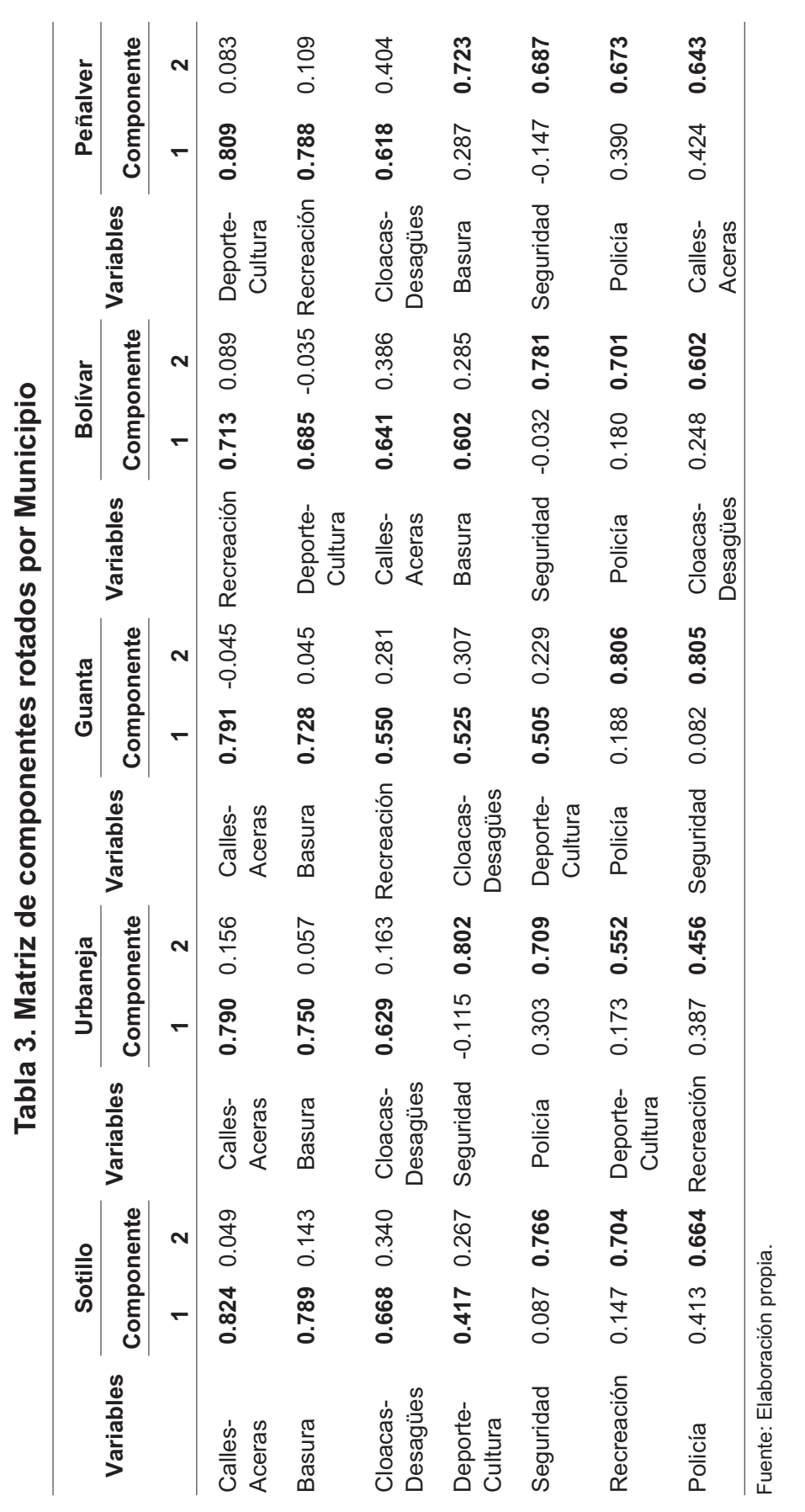


Análisis de la percepción de los servicios municipales en la comunidad anzoatiguense Núñez, Linda; Rodríguez, Daysi y Cáceres, Alfonso

Guanta priorizan los servicios de limpieza de aceras y calles y recolección de basura. Mientras que los municipios de Bolívar y Guanta le dieron mayor importancia a las políticas de recreación, cultura y deporte. Esto es refleja un sintoma claro de las prioridades de los servicios públicos en los municipios de la zona oriental de Anzoátegui y que han de ser considerados por los gerentes municipales.

La percepción de la comunidad en los servicios públicos, como parte de la participación ciudadana en las politicas públicas de la municipalidad, puede ser un claro control de las decisiones de las autoridades administrativas y además puede servir de apoyo a las decisiones de gestión gubernamental, tal como lo argumenta Fernández et al. (2008). Para llevar a cabo este cometido, el principal aporte de esta investigación es brindar a los entes gubernamentales(alcaldías) y a las comunidades organizadas (Consejos Comunales), una metodología de análisis de las políticas públicas en la implantación de los sistemas de control de gestión, indicadores, evaluación de políticas públicas; con el propósito ejecutar acciones a través de la participación ciudadana de lograr el bienestar de los habitantes de las comunidades locales. Finalmente, para futuras investigaciones, el estudio se pudiera extender hacia otros estados para hacer las comparaciones respectivas de acuerdo a las regiones y asi evidencia cuál es el comportamiento de la comunidad en cuanto a: cómo evalua la calidad de los servicos y a cuáles le da mayor importancia dependiendo del lugar donde se encuentra.

\section{Referencias Bibliográficas}

Acevedo, Diofante (2005). Nuevos enfoques del control de gestión y resultados, Alfpha Omega Estudio Creativo C.A., Caracas.

Anderson, J.A. (1996). Communication theory: epistemological foundations. New York, NY: The Guilford Press.

Blanco, María; Aibar, Beatriz y Aibar, Cristina (2007). "El papel de los indicadores de gestión en la implantación y evaluación de la filosofía de calidad en la administración local: estudio de un caso" en Revista de Estudios Politécnicos. Vol. V, No 8, 139-159.

Bossi, Alexandre (2001). "Control de gestión aplicado a las entidades públicas. Aspectos de las prácticas española y brasileña", en Contabilidad Internacional, http://www.5campus.com/leccion/congepub, 02-05-2009.

Bracho, Pedro (2001). La Democracia Participativa, I Congreso Iberoamericano de Estudiantes de Derecho. Ponencia en Maracaibo. Venezuela.

Cardozo, Myriam (2005). "Evaluación y participación social en el marco de la gerencia pública necesaria", en Revista Venezolana de Gerencia, v.10 n.29. enero, p.86-105.

Dunn, W. (2004). Public Policy Analysis: An Introduction, Third Edition, New Jersey: Prentice-Hall.

Escobar, Alejandro (2004). "Participación Ciudadana y Políticas Públicas. Una problematización acerca de la relación Estado y Sociedad Civil en América Latina en la última década", en Revista austral de ciencias sociales, Revista Electronica UACH, n.8 Valdivia p.97-108. Http://mingaonline.uach.cl/ scielo.php?script $=$ sci_arttext\&pid= S0718-17952004000100007\&lng=es \&nrm=iso, 28-04-2009. 
Fernández, Norcka; Belloso, Nora y Delgado, Fracys (2008). "Recursos informacionales, desarrollo endógeno y participación ciudadana en la Gestión Pública Local", en Revista Venezolana de Gerencia v.13 n.43 sep., p.377-397.

Gorsuch, R.L. (1997). "Exploratory factor analysis: its role in item analysis" en Journal of personality assessment, 68(3):532-60.

Guinart, Josep (2003). "Indicadores de gestión para las entidades públicas", VIII Congreso Internacional del CLAD sobre la reforma del Estado y de la Administración Pública. Panamá. 28 / 31 octubre 2003.

Lambin, J. (1995). Marketing estratégico, Segunda Edición, Mc Graw-Hill.

Langseth, Petter; Langan Patricia y Taliercio, Robert (1996). "Service Delivery Survey (SDS):A Management Tool”, en The Economic Development Institute World Bank, http://www4.worIdbank.org/afr/stats/pdf/sds.pdf, 2804-2009.

Marcano Yelitza, Ferrer Johanna y Talavera, Rosalba (2008). Gestión organizativa y operativa de los Consejos Comunales en el municipio Los Taques de la península de Paraguaná. Análisis de Coyuntura, Vol. 14, No. 2, p.173-181.

Matheus, María (2006). "Estado Federal Descentralizado Venezolano: Subsidiariedad y Relaciones Intergubernamentales", en Cuestiones Políticas, No.37, p. 131-152.

Montecinos, Egon (2006). "Descentralización y democracia en Chile: análisis sobre la participación ciudadana en el presupuesto participativo y el plan de desarrollo comunal", Revista de Ciencia Politica, Vol 26 No. 2, p. 191-208.

Morales, Eduviges; Núñez, Ingrid y Delfín, María (2006). "Repensando desde el plano normativo la participación ciu- dadana en la gestión pública", Revista Venezolana de Gerencia, Vol. 11 n.35, p. 453-470.

Núñez, Linda: Alfonso, Cáceres y Rodríguez, Daysi (2007). "Análisis Varimax de factores que influyen en la existencia de los niños de la calle según la opinión pública". Revista Saber, Vol. 19. No. 2, julio-diciembre, p. 240-246.

Oszlak, Oscar (2003). "El mito del Estado mínimo: una década de reforma estatal en la Argentina", en Desarrollo Económico. Revista de Ciencias Sociales, Vol. 42, $\mathrm{N}^{\circ} 168$, enero-marzo, pp. 519-543.

Pintaudi, Silvana (2005). "Participación Ciudadana En La Gestión Pública: Los Desafíos Políticos" en Revista Electrónica de Geografía y Ciencias Sociales, Vol. IX, núm. 194 (102). http://www.ub.es/geocrit/sn/sn-194102.htm, 28-04-2009.

Polino, Carmelo, Fazio, María Eugenia, Vaccarezza, Leonardo (2003). "Medir la percepción pública de la ciencia en los países iberoamericanos. Aproximación a problemas conceptuales". Revista Iberoamericana de Ciencia, Tecnología, Sociedad e Innovación. Número 5.

República Bolivariana de Venezuela (2006). Ley de Consejos Comunales, Publicada en Gaceta Oficial N ${ }^{\circ} \mathbf{5 . 8 0 6}$ (extraordinaria). Caracas.

República Bolivariana de Venezuela (2002). Ley de los Consejos Locales de Planificación Pública, Gaceta Oficial N³7.463, Caracas.

República Bolivariana de Venezuela (1999). Constitución de la República Bolivariana de Venezuela, Gaceta Oficial $N^{\circ} 36.860$, Caracas.

República Bolivariana de Venezuela (2005). Ley Orgánica del Poder Público Municipal, Gaceta Oficial N 38.204 . Caracas. 
Análisis de la percepción de los servicios municipales en la comunidad anzoatiguense Núñez, Linda; Rodríguez, Daysi y Cáceres, Alfonso

Robbins, S. (1998). Comportamiento organizacional. Octava Edición. Mc GrawHill.

Saltó, Esteve; Villalbí, Joan; Valverde, Araceli; Baranda, Lucia y Plasencia, Antoni (2006). "Políticas Reguladoras y Opinión Pública: El Caso del Tabaco" en Rev Esp Salud Pública, N. ${ }^{\circ} 3$, Mayo-Junio, 80: 243-248.

Sartori, Giovanni ( 2003). Elementos de Teoria Política. Alianza Editorial, Madrid.

Stanley, C.; lawrence, W.; James, E. (1999). Sensación y percepción. Tercera Edición. Mc Graw-Hill.
Weiss, Carol (1998). Evaluation Methods for Studying Programs and Policies. Second Edition, New Jersey: Prentice Hall.

Wittig, A. (1979). Introducción a la psicología, Primera Edición, Mc Graw-Hill.

White, Louise (1994). Values, Ethics, and Standards in Policy Analysis, 2da. Edition, Encyclopedia of Policy Studies. 